\title{
Growth and Detachment of Oxygen Bubbles Induced by Gold- Catalyzed Decomposition of Hydrogen Peroxide
}

\author{
Pengyu Lv, ${ }^{\dagger} \odot$ Hai Le The, ${ }^{\ddagger}$ Jan Eijkel,, Albert Van den Berg, ${ }^{\ddagger}$ Xuehua Zhang,, II, $\dagger \odot$
} and Detlef Lohse*,t,\$๑

\begin{abstract}
${ }^{\dagger}$ Physics of Fluids group, Faculty of Science and Technology, Max Planck - University of Twente Center for Complex Fluid Dynamics, MESA+ Institute, and J. M. Burgers Centre for Fluid Dynamics, and ${ }^{\ddagger}$ The BIOS Lab-on-a-Chip group, Faculty of Electrical Engineering, Max Planck - University of Twente Center for Complex Fluid Dynamics, Mathematics and Computer Science, MESA+ Institute, University of Twente, P. O. Box 217, 7500 AE Enschede, The Netherlands

${ }^{\text {II }}$ Soft Matter \& Interfaces Group, School of Engineering, RMIT University, Melbourne VIC 3001, Australia

${ }^{\S}$ Max Planck Institute for Dynamics and Self-Organization, 37077 Goettingen, Germany
\end{abstract}

\section{Supporting Information}

ABSTRACT: Whereas bubble growth out of gas-oversatured solutions has been quite well understood, including the formation and stability of surface nanobubbles, this is not the case for bubbles forming on catalytic surfaces due to catalytic reactions, though it has important implications for gas evolution reactions and self-propulsion of micro/ nanomotors fueled by bubble release. In this work we have filled this gap by experimentally and theoretically examining the growth and detachment dynamics of oxygen bubbles from hydrogen peroxide decomposition catalyzed by gold. We measured the bubble radius $R(t)$ as a function of time by confocal microscopy and find $R(t) \propto t^{1 / 2}$. This diffusive growth behavior demonstrates that the bubbles grow from an oxygenoversaturated environment. For several consecutive bubbles detaching from the same position in a short period of time, a well-repeated growing behavior is obtained from which we conclude the absence of noticeable depletion effect of oxygen from previous bubbles or increasing oversaturation from the gas production. In contrast, for two bubbles far apart

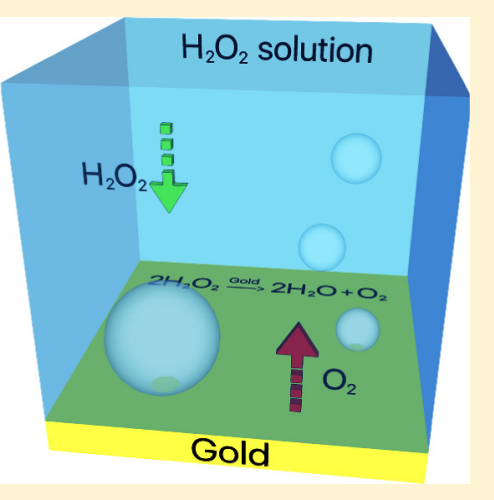
either in space or in time, substantial discrepancies in their growth rates are observed, which we attribute to the variation in the local gas oversaturation. The current results show that the dynamical evolution of bubbles is influenced by comprehensive effects combining chemical catalysis and physical mass transfer. Finally, we find that the size of the bubbles at the moment of detachment is determined by the balance between buoyancy and surface tension and by the detailed geometry at the bubble's contact line.

\section{INTRODUCTION}

The dynamics of nucleation, growth, and detachment of gas bubbles has important implications for many catalytic reactions which include gas formation. On one hand, the burst and detachment of bubbles may enhance the flow and mass transfer for the reactions. ${ }^{1,2}$ The microbubble dynamics has also been exploited to measure local surface tension or to create autonomous motion of the catalytic particles as micromotors or nanorockets. ${ }^{3-7}$ In a typical example, ${ }^{8-11}$ released bubbles propel a directional movement of micromotors, which is fueled by the reaction $2 \mathrm{H}_{2} \mathrm{O}_{2} \stackrel{\text { catalyst }}{\longrightarrow} 2 \mathrm{H}_{2} \mathrm{O}+\mathrm{O}_{2}$, catalyzed by a metal catalyst such as platinum, silver, and gold. On the other hand, bubbles attached to the catalyst surface hinder the direct contact of the catalyst to reactants in the solution, impeding the catalytic reaction. ${ }^{12,13}$ Cavitation induced by the bubble collapse close to the catalysts may also damage the catalytic surfaces. $^{14}$

How to control and efficiently remove the bubbles from the catalytic surface or even suppress their formation is thus an important topic for the design of gas-evolving reactions.
However, hitherto the focus in the literature has been on bubble nucleation and growth out of gas-oversaturated solutions, without chemical reactions. Then the bubble nucleation is always heterogeneous; ${ }^{15-18}$ i.e., at some surface, and thanks to pinning forces even diffusively stable surface nanobubbles can form. ${ }^{19}$ On the larger microscale and beyond, such a stabilization is not possible and such bubbles will in general diffusively grow in gas-oversaturated solutions. For isolated bubbles in an unbound environment, the diffusive growth behavior can even analytically be calculated. ${ }^{20-22}$ For single bubbles in gas-oversatured solution, also the influence of a neighboring wall to which the bubble is attached, the effect of density-driven convection, the ultimate size at which detachment takes place, the effect of prior bubbles (i.e., "history effects"), and other complications have been reasonably well understood, see, e.g., refs 23-30.

Received: May 23, 2017

Revised: September 1, 2017

Published: September 5, 2017 
(a)

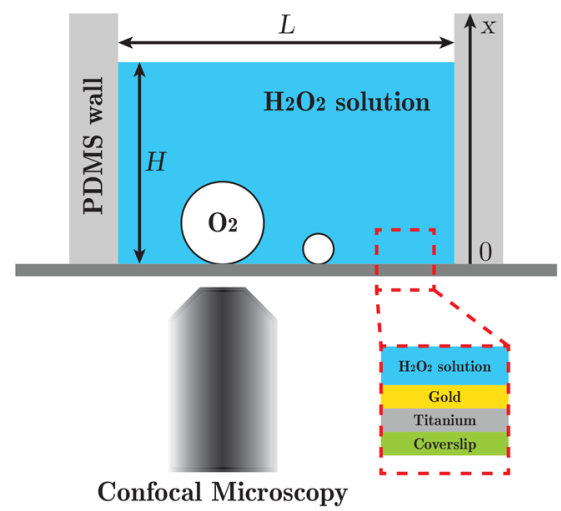

(b)

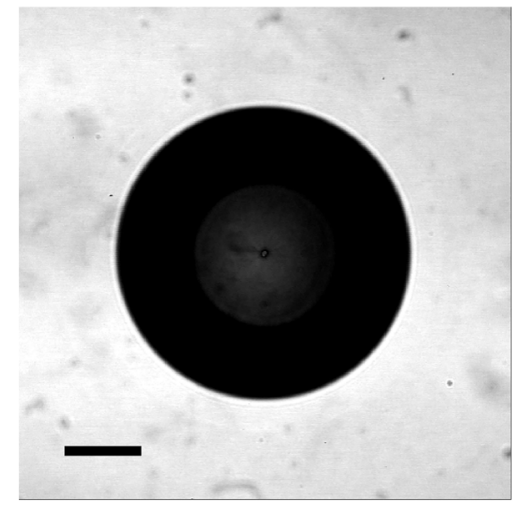

(c)

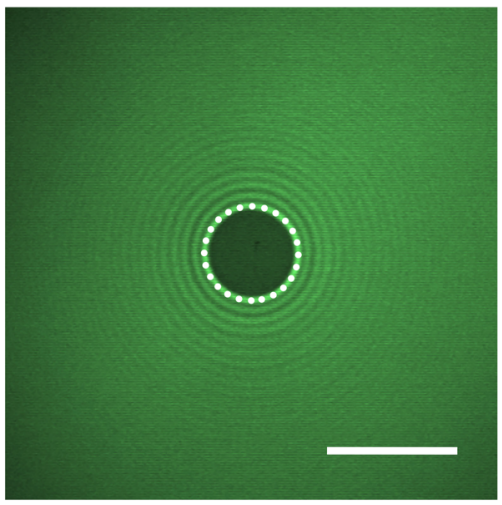

Figure 1. (a) Schematics of the experiment setup. A glass substrate with a thickness of $170 \mu \mathrm{m}$ is coated with a $23 \mathrm{~nm}$ thick gold-titanium binary layer, on which gold is exposed to $\mathrm{H}_{2} \mathrm{O}_{2}$ solution and acts as the catalyst for the decomposition process. A PDMS container is filled with $\mathrm{H}_{2} \mathrm{O}_{2}$ solution with a depth of $H=5 \mathrm{~mm}$. The area of the reacting interface is $A=L \times L$, where $L=10 \mathrm{~mm}$. The growth of oxygen bubbles is measured by confocal microscopy. (b) Representative confocal image of a growing bubble. The focal plane is located at the bubble bottom, namely, the reacting interface. The transmitted image is actually the projection of the bubble on the transmitted light detector, from which the bubble radius can be measured as a function of time. The scale bar is $200 \mu \mathrm{m}$. (c) Reflective image of the bubble base area. The bubble is pinning at a contact line indicated by the dotted line, based on which the effective base radius $R_{\mathrm{p}}$ can be calculated. The Newton's rings induced by the interference of lights from the liquid-solid interface and liquid-gas interface clearly demonstrate the pinning region. The scale bar is $20 \mu \mathrm{m}$.

However, all this does not hold for bubbles forming out of catalytic reactions. Indeed, in contrast to bubbles simply forming out of oversaturated solutions, the bubble growth dynamics in catalytic reactions emerges from an interplay of the reaction kinetics and the physical mass transfer, and a quantitative understanding of the coupled effects from the reaction and the gas diffusion is still lacking. In this work, we will therefore experimentally and theoretically investigate the growth and detachment process of the bubbles from the catalytic decomposition of hydrogen peroxide.

Our model system consists of a horizontal flat catalyst surface in an aqueous solution of hydrogen peroxide. Gold was selected to be the catalyst because its relatively high activation energy enables regulating the bubble growth rate to be suitable for experimental observation. In the experiments, a complete lifecycle of the bubble evolving at the interface of the gold catalyst and the solution goes through the phases of nucleation, growth, and detachment.

\section{EXPERIMENTAL SECTION}

The experimental setup is shown in Figure 1a. A $170 \mu$ m thick coverslip was continuously coated with a thin gold-titanium binary layer possessing a thickness of $20 \mathrm{~nm}$ plus $3 \mathrm{~nm}$, referring to the gold and the titanium films, respectively. Gold acted as the catalyst for the decomposition of hydrogen peroxide, and titanium was used to strengthen the binding performance between the gold film and the glass substrate in order to avoid any delamination during bubble formation. In order to obtain a smooth uniform coating, the glass coverslip was successively immersed in a 99\% nitric acid $\left(\mathrm{HNO}_{3}\right)$ solution for $5 \mathrm{~min}$, and in a $69 \% \mathrm{HNO}_{3}$ solution at $95{ }^{\circ} \mathrm{C}$ for 10 $\mathrm{min}$, followed by rinsing with deionized water in a quick dump tank and drying thoroughly in a nitrogen flow. The goldtitanium binary layer was deposited on the coverslip using an ion-beam sputtering system (home-built T'COathy machine, NanoLab, University of Twente). All the sputtering processes were done at a pressure of $6.6 \times 10^{-3} \mathrm{mbar}$ and a power of 200 $\mathrm{W}$. The process pressure was adjusted by an argon flow.
A square PDMS (poly(dimethylsiloxane), with a curing ratio of 10:1) wall with an inner side length of $L=10 \mathrm{~mm}$ and a height of $10 \mathrm{~mm}$ was cast to construct a container of solution by adhering to the substrate. Hydrogen peroxide aqueous solution, $30 \%(\mathrm{w} / \mathrm{w}),\left(\mathrm{H}_{2} \mathrm{O}_{2}\right.$, Sigma-Aldrich) was used as received, and the depth of the solution in the PDMS container was $H=5 \mathrm{~mm}$ which was large enough compared with the detaching bubble diameter (smaller than $1 \mathrm{~mm}$, see Figure 5a).

Real-time optical observation was carried out to monitor the bubble growth using an inverted laser scan confocal microscope (Nikon confocal microscope Al system, Nikon Corporation, Tokyo, Japan) with a $10 \times$ dry objective (CFI Plan Fluor $10 \times$ / 0.3 DIC, numerical aperture $(\mathrm{NA})=0.3$, working distance $(\mathrm{WD})=16 \mathrm{~mm})$. Compared with a normal optical microscope, a confocal microscope can eliminate the out-of-focus lights by crucially adding a pinhole on the confocal plane of the lens which acts as a filter of lights from the focal plane. In the current experiments, unless otherwise noted, the microscope was constantly focused on the gold-solution interface, namely the bubble bottom, where reaction took place. A laser with a wavelength of $561 \mathrm{~nm}$ was used to observe the bubble formation from below as shown in Figure 1a. A part of the laser lights can transmit through the substrate to get to the transmitted light detector as the gold-titanium binary layer coated on the coverslip was so thin $(23 \mathrm{~nm}$ in total) that the substrate was still transparent for visible lights, while the other part of lights reflects at the gold-solution interface back to the spectral detector. Therefore, we combined two channels, i.e., transmission channel and reflection channel, to observe the projection of the bubble (transmitted image, Figure $1 \mathrm{~b}$ ) and the bubble base area (reflective image, Figure 1c) simultaneously.

The continuous decomposition of hydrogen peroxide was performed over the entire reacting interface to achieve a highly oxygen-oversaturated solution after the catalytic reaction, leading to the formation of bubbles everywhere at the beginning of the experiments. However, after $1 \mathrm{~h}$ growth, coalescence, and detachment, bubbles preferred to nucleate at some preferred sites, like impurities and defects. Then also successive bubbles nucleated at the same sites as that of the 
(a)

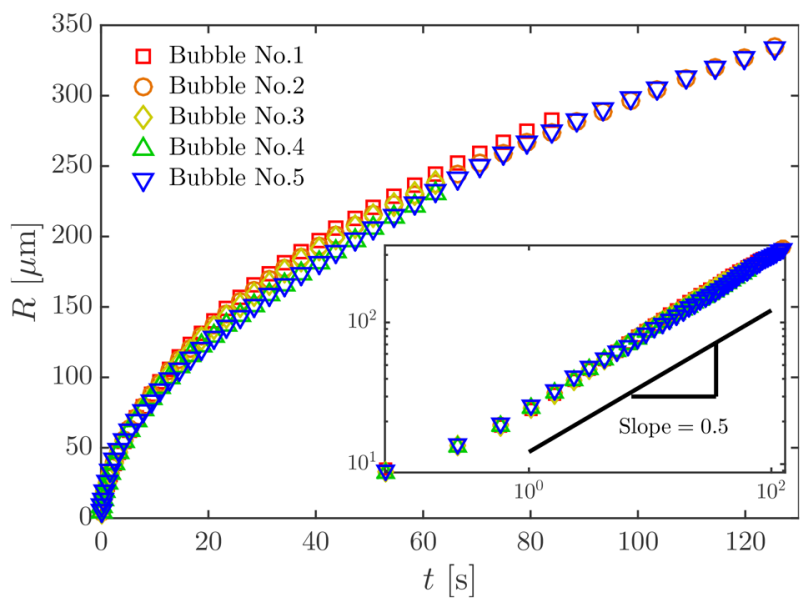

(b)

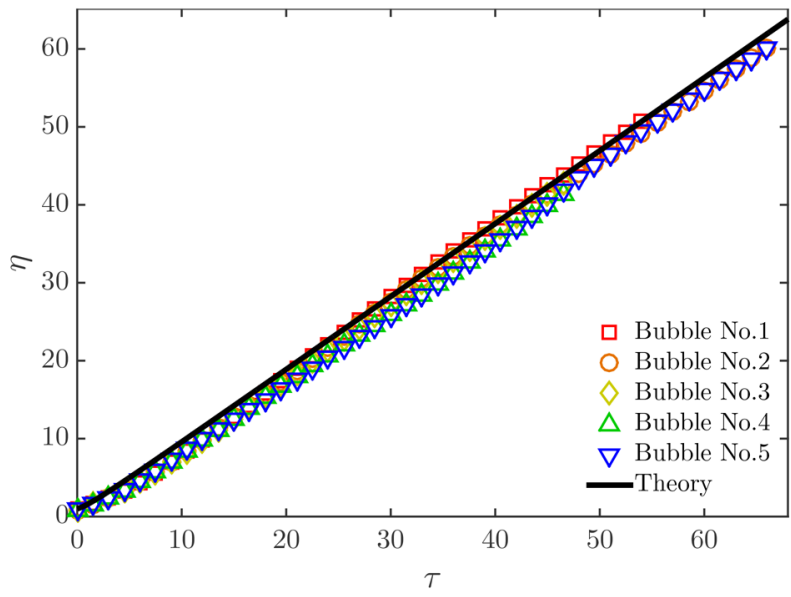

(c)

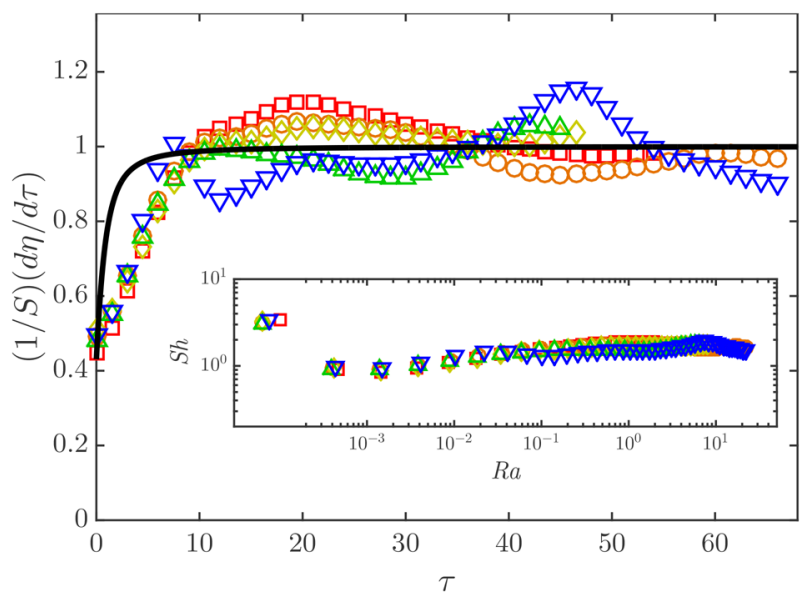

Figure 2. Growth of consecutive bubbles at the identical position showing the variations of radius (a) and in (b) dimensionless form $\eta(\tau)$ and its derivative (c) with respect to $\tau \propto \sqrt{ }$ t. Symbols represent the experimental data of five bubbles spanning 8 min, while the solid line in (b) and (c) represents the full analytical solution to eq 5 and its normalized derivative, respectively. The fitted oversaturation for the theoretical curve is $\zeta=8$. The inset in (a) shows that the radius scales with time as $R(t) \propto t^{1 / 2}$. The fluctuations of the data in (c) correspond to oscillations of the surrounding solution. The inset in (c) shows the Sherwood number as a function of the mass transfer Rayleigh number. The constant Sherwood number implies a pure diffusive mass transfer process.

previously detached ones. Thus, we were able to observe the bubble growth on several different fixed spots with a certain distance in between. In the course of the data collections, which was $1 \mathrm{~h}$ later than the beginning of the experiments, no direct contact took place between any two bubbles before detachment. Every set of experiment took $10 \mathrm{~min}$ at most and included four to seven consecutively growing bubbles, in which the number of bubbles depended on the local situations that the bubbles encountered, such as the local concentration gradient and the detaching bubble size. The scanning frame rate was $30 \mathrm{~s}^{-1}$ in resonant mode. All the experiments were operated at a constant temperature of $23^{\circ} \mathrm{C}$.

Figures $1 \mathrm{~b}$ and $1 \mathrm{c}$ show representative confocal images of $\mathrm{a}$ growing bubble. The transmitted image (Figure $1 \mathrm{~b}$ ) presents the bubble morphology which reveals the variation of bubble radius $R$ as a function of time. The reflective image (Figure 1c) zooms into the bubble base area, revealing the pinning contact line indicated by the dotted line. The effective bubble base pinning radius $R_{\mathrm{p}}$ can be calculated by equating $\pi R_{\mathrm{p}}^{2}$ to the measured area of the pinning region (the area enclosed by the dotted line in Figure 1c).

\section{RESULTS AND DISCUSSION}

We first address the growth of the consecutive oxygen bubbles at the identical location from which the previous one has just detached. The time interval between two consecutive bubble detachments is always less than $10 \mathrm{~min}$. We employed confocal microscopy to observe and record the growth of the bubbles by measuring the bubble radius $R$ as a function of time $t$. Figure $2 \mathrm{a}$ shows the results of five consecutive bubbles growing at the same position spanning $8 \mathrm{~min}$. The logarithmic plot in the inset of Figure 2a reveals that the radius scales with time as $R(t) \propto$ $t^{1 / 2}$, which is consistent with previous results on bubble growth in oversaturated solution without a catalytic gas production reaction. ${ }^{20,21,26}$ In Figure $2 \mathrm{~b}$, all experimental data have been converted into the dimensionless radii $\eta=R / R_{\mathrm{p}}$, where $R_{\mathrm{p}}$ is the effective radius of the pinning contact area and $\tau \propto \sqrt{t}$ is employed to be indicative of the $t^{1 / 2}$ dependence of $R(t)$ where the exact definition of $\tau$ is given later in eq 6 . The plot shows that $\eta$ increases proportionally to $\tau$. The experimental data of five consecutive bubbles follow an identical pattern, exhibiting a repeated bubble growing behavior. Note that six independent experiments have been performed to verify the correctness in 
statistics. Each set of experiment contains at least four consecutive bubbles. A good repeatability of experimental results is obtained (see the Supporting Information).

As shown in Figure $2 c$, the dimensionless growth rates $d \eta / d \tau$ increase quickly and converge to a plateau value eventually, which shows a consistent value between different bubbles, especially for the early accelerating growth stages. No delay in growth was observed for newly formed bubbles as compared to prior ones in reaching the plateau value for the given period of reaction time. The consistency in the early growth of consecutive bubbles suggests that the oxygen depletion due to the growth and detachment of the previous bubbles has negligible influence on the subsequent bubbles in the current situations. In contrast, for bubble growth out of oversaturated solution, a delayed growth had been observed due to gas depletion in the surroundings of the bubble..$^{23,28,31,32}$ The identical growth rate of consecutive bubbles suggests that the gas oversaturation has reached dynamic equilibrium; that is, the production of the gas from the reaction reaches a balance with the gas supplied to the bubble growth. Here, the gas oversaturation is denoted as $\zeta=C_{\infty} / C_{s}-1$, where $C_{\infty}$ is the gas concentration far from the bubble and $C_{\mathrm{s}}$ is the saturation concentration.

We next compare the bubbles either far apart in space or with long time interval in between (farther than $5 \mathrm{~mm}$ in space distance or longer than $1 \mathrm{~h}$ in time interval). The dimensionless radii $\eta$ as a function of $\tau$ are plotted in Figure 3. Bubble A, the reference bubble, formed at position No. 1 and started to grow at the time of $t=2 \mathrm{~h}, 45 \mathrm{~min}$ later than the start of the experiment. Bubble $\mathrm{B}$, formed not long before the formation of bubble $A$ at the time $t=2 \mathrm{~h}, 41 \mathrm{~min}$, was located $5 \mathrm{~mm}$ away from bubble A. Bubble $\mathrm{C}$ was at the same position as bubble $\mathrm{A}$ but started to grow at the time $t=4 \mathrm{~h}, 27 \mathrm{~min}$, nearly $2 \mathrm{~h}$ later than bubble A. Unlike the repeated growth of the closely consecutive bubbles, substantial discrepancies exist in the growth of these three bubbles as shown in Figure 3a. Bubble B grew apparently faster than bubble $\mathrm{A}$, whereas bubble $\mathrm{C}$ exhibited a slower growth rate. The difference in the growth rate of the bubbles suggests differences in local oxygen oversaturation around each bubble, which will be discussed below in detail to reveal the mechanism.

We now analyze whether the extent of reaction and the distribution of $\mathrm{H}_{2} \mathrm{O}_{2}$ are reaction-limited or diffusion-limited, which is highly related to the production and distribution of $\mathrm{O}_{2}$ as well as the growth of bubbles. The (dimensionless) Damköhler number $D a$ is defined as ratio of the characteristic diffusion time and the characteristic reaction time. It reflects the effect of the diffusive mass transfer on the chemical reaction process

$$
D a=\frac{t_{\mathrm{d}, \mathrm{H}_{2} \mathrm{O}_{2}}}{t_{\mathrm{r}}}=\frac{H k}{\pi D_{\mathrm{H}_{2} \mathrm{O}_{2}}}
$$

Here $t_{\mathrm{r}}$ is characteristic reaction time, $t_{\mathrm{d}}$ is characteristic diffusion time, $H$ is the characteristic length (taken to be the depth of the solution), $D_{\mathrm{H}_{2} \mathrm{O}_{2}}$ is the diffusion coefficient of $\mathrm{H}_{2} \mathrm{O}_{2}$, and $k$ is the reaction rate constant. For large $\mathrm{Da} \gg 1$, the reaction is much faster than the diffusion, denoting a diffusionlimited process in which the diffusion dominates and the reaction is regarded to be instantaneously in equilibrium, whereas for small $D a \ll 1$, the process is reaction-limited. In our experiments, $D a \approx 29$, suggesting that the extent of reaction is dominated by diffusion, which also determines the (a)

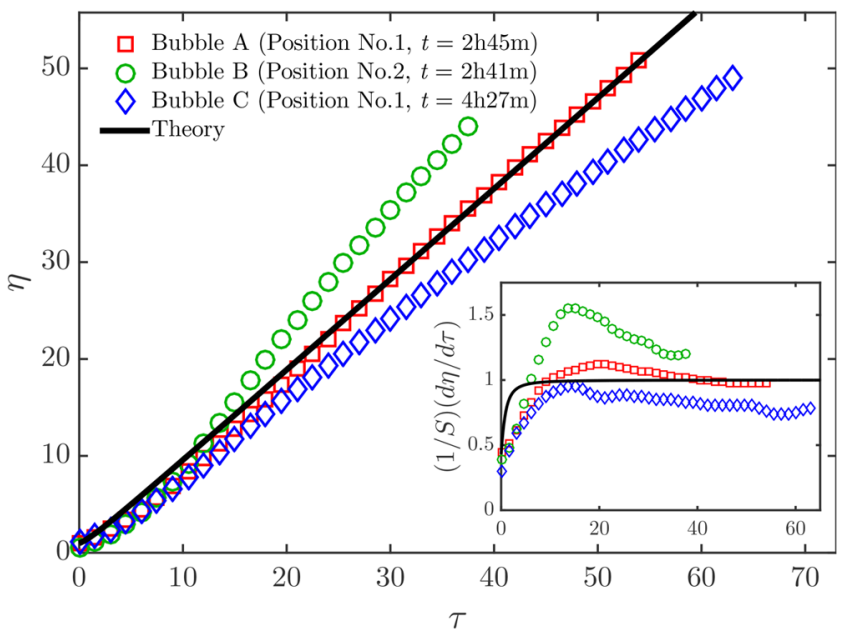

(b)

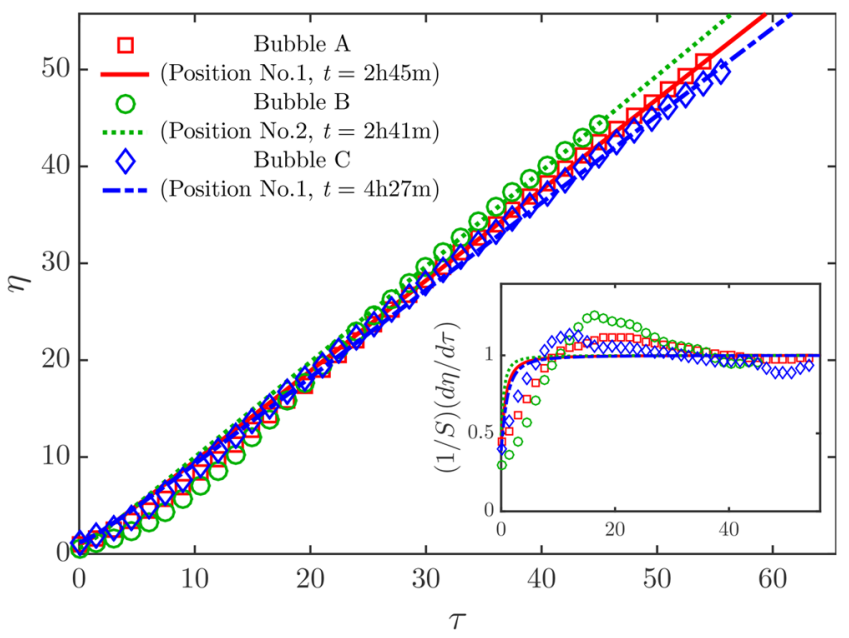

Figure 3. (a) Discrepant growth of bubbles located far away from each other and bubbles forming far apart in time. Time $t$ is counted since the substrate is exposed to the solution in the experiment. Bubble A (square symbol) in the position No. 1 is the reference bubble. Bubble $\mathrm{B}$ (circle symbol) grows at the time close to bubble A but located at the position (No. 2) $5 \mathrm{~mm}$ away from bubble A. Bubble C (diamond symbol) formed at the same location as bubble A but grows nearly $2 \mathrm{~h}$ later than bubble $\mathrm{A}$. The data reveal discrepancies in the growth rates, also reflected in the different plateau values of the data curves in the inset. The solid lines are the analytical solution of bubble $\mathrm{A}$, assuming $\zeta=8$. (b) Recast data of bubbles B and $\mathrm{C}$ with $\zeta_{\mathrm{B}}=11.3$ and $\zeta_{\mathrm{C}}=6$, respectively, reveal the different concentration gradients to which the bubbles are subject. The data correspondingly collapse well onto the corrected analytical solutions as shown by the lines.

distribution of $\mathrm{H}_{2} \mathrm{O}_{2}$ across the depth of the solution. The detailed calculation of $\mathrm{Da}$ is provided in the Supporting Information.

Here we note that this diffusion-limited process should also hold for most of the common catalysts, like platinum, silver, palladium, and cobalt-iron oxides. These catalysts possess lower activation energies than gold, ${ }^{33-36}$ indicating a faster reaction rate and thus a larger $\mathrm{Da}$. Indeed, taking platinum for example, our experiments show that only $2.5 \%$ (w/w) $\mathrm{H}_{2} \mathrm{O}_{2}$ solution in contact with platinum film generated much more bubbles growing in a much more violent way than in the situation with gold exposed to $30 \%(w / w) \mathrm{H}_{2} \mathrm{O}_{2}$ solution as 
reported here. Therefore, the dynamics for most of the common catalysts are expected to be the same as the one in the current gold case, as long as $D a>1$. However, because of the slow chemical reaction rate, gold with higher activation energy is an ideal catalyst for the investigations of bubble formation due to the decomposition of $\mathrm{H}_{2} \mathrm{O}_{2}$.

The concentration profiles across the depth of the liquid for both $\mathrm{H}_{2} \mathrm{O}_{2}$ and $\mathrm{O}_{2}$ over $5 \mathrm{~h}$ are shown in Figure 4 (see the

(a)

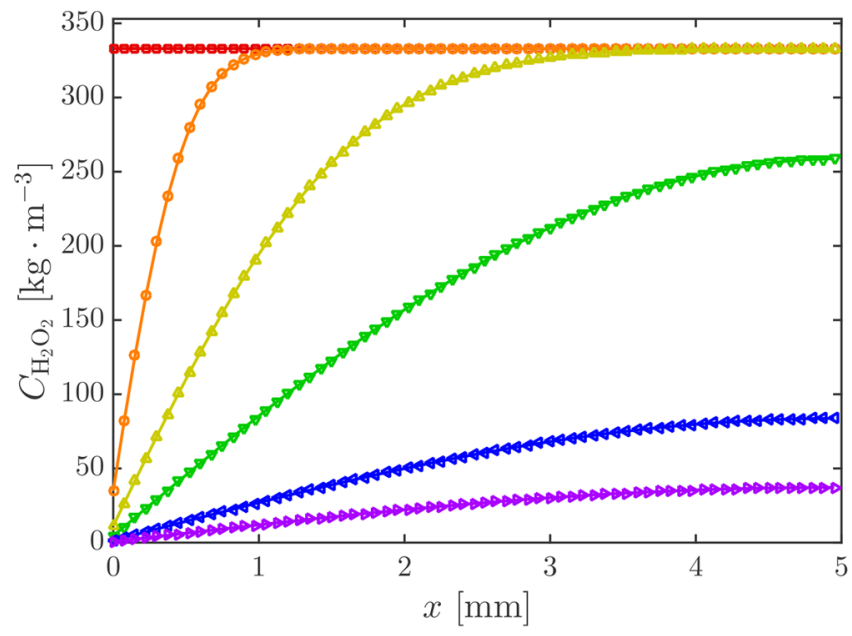

(b)

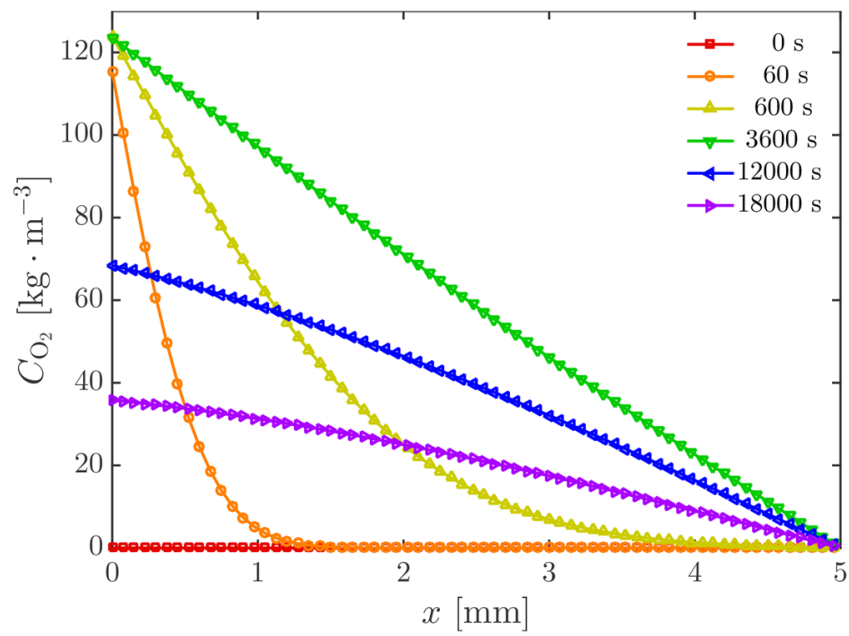

Figure 4. Concentration profiles across the depth of the solution for both $\mathrm{H}_{2} \mathrm{O}_{2}$ (a) and $\mathrm{O}_{2}$ (b) over $5 \mathrm{~h}$ considering a pure diffusion process without the bubble growth. In (a), an obvious depletion of $\mathrm{H}_{2} \mathrm{O}_{2}$ is obtained due to the catalytic reaction at the reacting interface $(x=0)$. In (b), a rapid increase of the oxygen concentration near the reacting interface is achieved at the beginning of the reaction, indicating a highly oxygen-oversaturated state due to the accumulation of $\mathrm{O}_{2}$ molecules. As the reaction continues, the oxygen concentration decreases, which implies an expiration of the catalytic reaction.

Supporting Information for details of how the profiles are calculated). Although the initial consumption rate of $\mathrm{H}_{2} \mathrm{O}_{2}$ is quite fast near the reacting interface, the slow depletion of $\mathrm{H}_{2} \mathrm{O}_{2}$ as shown in Figure $4 \mathrm{a}$ reveals the diffusion-limited process. Meanwhile, a rapid initial increase of the oxygen concentration is obtained near the reacting interface as shown in Figure $4 \mathrm{~b}$, leading to the accumulation of $\mathrm{O}_{2}$ molecules which results in a highly oxygen-oversaturated state. (Note that
$C_{\infty}$ actually refers to the local oxygen concentration near $x=0$ where the bubble grows.) This diffusive behavior of oxygen has a great effect on the bubble growth.

Moreover, the oxygen-supersaturated state can also be examined by the ratio between the production rate and the diffusion rate $t_{\mathrm{d}, \mathrm{O}_{2}} / t_{\mathrm{r}}=H k /\left(\pi D_{\mathrm{O}_{2}}\right) \approx 19 \gg 1$, where $D_{\mathrm{O}_{2}}$ is the diffusion coefficient of $\mathrm{O}_{2}$. The fact that the reaction occurs much faster than the diffusion means that oxygen oversaturation can easily be achieved (see Figure 4b), which allows for the nucleation and the growth of new bubbles. Eventually, a dynamical balance between the consumption rate of the gas by the bubble growth and the replenishment rate of the gas from the reaction is reached. Therefore, the oversaturation for the growth of consecutive bubbles is at the same level.

To reveal the diffusion-dominated feature in the bubble growth, we recast the experimental data of Figure 2 in terms of the Sherwood and the Rayleigh numbers

$$
\begin{gathered}
S h=\frac{2 R h}{D_{\mathrm{O}_{2}}}=\frac{2 \rho_{\mathrm{G}} R \dot{R}}{\zeta C_{\mathrm{s}} D_{\mathrm{O}_{2}}} \\
R a=\frac{g \lambda_{\mathrm{C} \zeta C_{\mathrm{s}}(2 R)^{3}}}{\nu D_{\mathrm{O}_{2}}}
\end{gathered}
$$

where $h=\rho_{\mathrm{G}} \dot{R} /\left(\zeta C_{\mathrm{s}}\right)$ is the mass transfer coefficient, $\rho_{\mathrm{G}}=p_{0} M / \mathcal{R} T=1.3169 \mathrm{~kg} \cdot \mathrm{m}^{-3}$ is the oxygen density inside the bubble, $p_{0}=1.01325 \times 10^{5} \mathrm{~Pa}$ is the atmospheric pressure, $M=0.032 \mathrm{~kg} \cdot \mathrm{mol}^{-1}$ is the molar mass of oxygen, $\mathcal{R}=8.314 \mathrm{~J} \cdot \mathrm{K}^{-1} \cdot \mathrm{mol}^{-1}$ is the universal gas constant, $T=$ $296.15 \mathrm{~K}$ is the temperature, $g$ is the acceleration of gravity, $\lambda_{C}$ $=4.7 \times 10^{-5} \mathrm{~m}^{3} \cdot \mathrm{kg}^{-1}$ is the concentration expansion coefficient of the solution, ${ }^{37}$ and $\nu=1.0 \times 10^{-6} \mathrm{~m}^{2} \cdot \mathrm{s}^{-1}$ is the kinematic viscosity. (We adopt the empirical formula for the variation of the solution density as a function of the oxygen concentration in ref 37, namely, $\rho_{s}=\{[0.0311+0.00154(T-273.15)] \times$ $\left.10^{-3}\right\} C_{\mathrm{O}_{2}} V_{\mathrm{m}} / M+\rho_{\mathrm{H}_{2} \mathrm{O}}$, where $\rho_{\mathrm{s}}$ is the density of the solution, $C_{\mathrm{O}_{2}}$ is the oxygen concentration, $V_{\mathrm{m}}$ is the molar volume of the ideal gas at the atmospheric pressure, and $\rho_{\mathrm{H}_{2} \mathrm{O}}$ is the water density. Therefore, $\lambda_{\mathrm{C}}=\left(\partial \rho_{s} / \partial \mathrm{C}_{\mathrm{O}_{2}}\right) / \rho_{\mathrm{H}_{2} \mathrm{O}}=4.7 \times 10^{-5} \mathrm{~m}^{3}$. $\mathrm{kg}^{-1}$.) We replot the data in the inset of Figure $2 \mathrm{c}$ and for large $\mathrm{Ra}$ obtain a constant Sherwood number, suggesting a purely diffusive mass transfer process during the growth of bubbles. No density-driven convection is developing around the bubbles, as was observed for the growth of $\mathrm{CO}_{2}$ bubbles out of $\mathrm{CO}_{2}$ oversaturated solution, ${ }^{23}$ as there the $\mathrm{CO}_{2}$-saturated water is heavier than pure water.

Next we will even quantitatively explain the growth rate of consecutive bubbles and of bubbles far apart in space or in time. We consider the diffusive growth of a bubble on an impermeable substrate surrounded by a gas-oversaturated solution. As the bubble grows, a diffusion boundary layer with thickness of $\delta=\sqrt{\pi D_{\mathrm{O}_{2}} t}$ also grows outward from the bubble surface, across which there is a concentration gradient from $C_{\infty}$ to $C_{s}$. According to Henry's law, $C_{s}=H^{\mathrm{cp}} p_{\mathrm{G}}$, where $H^{\mathrm{cp}}=4.16 \times 10^{-7} \mathrm{~kg} \cdot \mathrm{m}^{-3} \cdot \mathrm{Pa}^{-1}$ is Henry's constant for oxygen in water at room temperature ${ }^{38}$ and $p_{\mathrm{G}}$ is the gas pressure inside the bubble. In our system, the Laplace pressure can be neglected throughout as the influence of the surface tension is limited to the very beginning of growth. Hence, $C_{s}=H^{\mathrm{cp}} p_{0}$ is constant. 
(a)

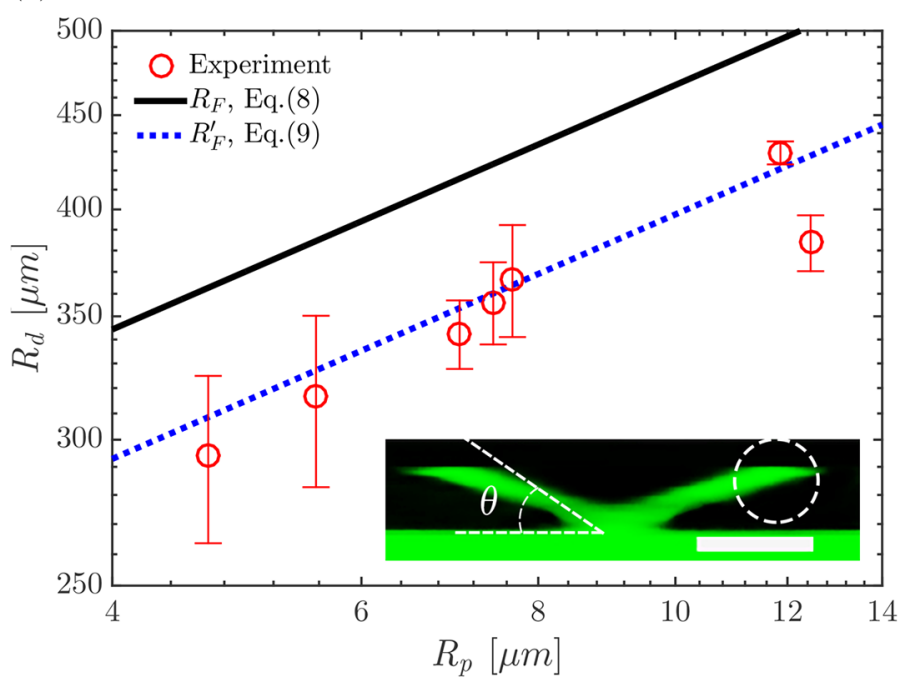

(b)

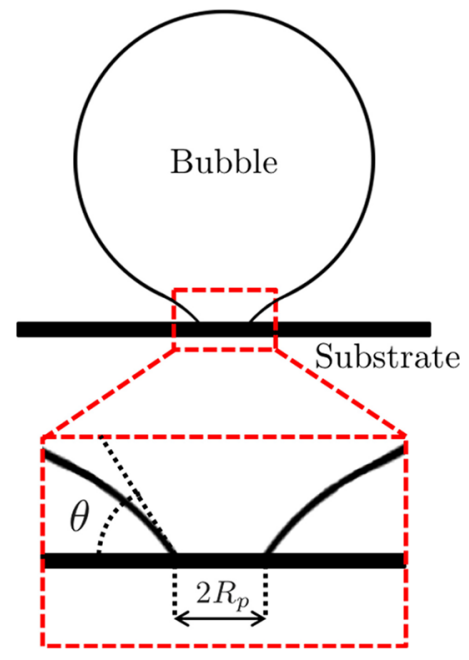

Figure 5. (a) Bubble radius $R_{\mathrm{d}}$ at the moment of detachment as a function of the effective bubble base radius $R_{\mathrm{p}}$. The black solid line displays the prediction for the Fritz radius $R_{\mathrm{F}} \propto R_{\mathrm{p}}^{1 / 3}$, eq 8 . The revised Fritz prediction, i.e., eq 9 (blue dotted line), taking into account the influence of the contact angle $\theta$ which is defined as the angle of the local liquid-gas interface inclined on the substrate as shown in the inset, has a better agreement with the experimental data than does eq 8 . The confocal image in the inset shows the morphology of the root portion of the bubble immediately before the detachment. One clearly sees that the bubble has already lifted upward away from the substrate by buoyancy. The pinning of the contact line allows the surface tension to resist the buoyancy to postpone the detachment, eventually leading to a necking stretch distortion of the bubble root. Because the scan direction is from bottom to top, the vanishing of the reflection signal in the dashed circle indicates that detachment has occurred. The scale bar is $5 \mu \mathrm{m}$. A movie showing the shape distortion of the bubble surface is included in the Supporting Information. (b) Sketch of bubble at the moment of detachment illustrating the definitions of $R_{\mathrm{p}}$ and $\theta$.

We also take into account the blocking effect of mass transfer due to the presence of the substrate. ${ }^{23}$ The mass transfer is assumed to take place over the effective area of the bubble surface. Therefore, the quasi-static radial growth rate is expressed as

$$
\frac{\mathrm{d} R}{\mathrm{~d} t}=\frac{\zeta C_{\mathrm{s}} D_{\mathrm{O}_{2}}}{\rho_{\mathrm{G}}}\left(\frac{1}{2 R}+\frac{1}{\sqrt{\pi D_{\mathrm{O}_{2}} t}}\right)
$$

It is convenient to express eq 4 in dimensionless form

$$
\frac{\mathrm{d} \eta}{\mathrm{d} \tau}=\frac{\tau}{2 \eta}+\sqrt{\frac{2 \zeta \mathrm{C}_{\mathrm{s}}}{\pi \rho_{\mathrm{G}}}}
$$

with $\eta=R / R_{\mathrm{p}}$ and

$$
\tau=\sqrt{\frac{2 \zeta C_{s} D_{\mathrm{O}_{2}}}{\rho_{\mathrm{G}} R_{\mathrm{p}}^{2}} t}
$$

We can solve this ordinary differential equation (ODE) to obtain the full solution with an initial condition $\eta(0)=R(0) /$ $R_{\mathrm{p}}$, where $R(0)$ is the initial radius of each individual bubble read from experimental data. Besides, when $\eta \gg 1$ and $\tau \gg 1$, there is an asymptotic solution to the $\mathrm{ODE}^{19,20}$

$$
\eta \approx\left(\sqrt{\frac{\zeta C_{\mathrm{s}}}{2 \pi \rho_{\mathrm{G}}}}+\sqrt{\frac{1}{2}+\frac{\zeta C_{\mathrm{s}}}{2 \pi \rho_{\mathrm{G}}}}\right) \tau \equiv S \tau
$$

The above theoretical prediction eq 5 for the growth of bubbles is plotted in Figure 2 and Figure 3 together with the experimental data. Here, the oversaturation $\zeta$ is not a priori known and must be adjusted to the experimental data. We find that after some transient, $\zeta=8$ gives a good description of the data. The derivative of the full solution in Figure $2 c$, i.e., $d \eta / d \tau$, is normalized by the asymptotic value of the derivative $S$, i.e., the terms inside the brackets in eq 7 . For the repeated growth of the closely consecutive bubbles in Figure 2, the oversaturation $\zeta=8$ not only describes the first bubble but also the later ones which follow immediately.

In the very early phase of the bubble's life, the theoretical curve shows faster growth (i.e., a larger rate) than the experimental data. This transient behavior can be explained as follows: In the theoretical analysis, the thickness of the diffusion boundary layer is initially assumed to be $\delta=0$, growing proportionally to $\sqrt{D_{\mathrm{O}_{2}} t}$. This theoretical assumption is not realistic for our experiments, in which due to prior equilibration, the diffusion boundary layer may have already reached a certain thickness during the interval between two consecutive bubbles, which results in a smaller concentration gradient and hence a slower growth rate than the theoretical predication. Moreover, there is a geometry factor for the bubble, which grows in the shape of a spherical cap. In contrast, the theoretical analysis only considers the bubbles as a full sphere, which causes a relatively large error for the bubbles in their initial phase, in which they are spherical cap shaped. ${ }^{23}$ For such reasons, a lower growth rate in the early stage of the growth is expected in the experimental data, as indeed seen in the experimental results (Figure 2c).

Next, to address and describe the discrepant growth rates of the bubbles being far apart from bubble $A$ either in space or in time, we must adjust the oversaturation to $\zeta_{B}=11.3$ and $\zeta_{C}=6$ for bubble $\mathrm{B}$ and $\mathrm{C}$, respectively, to agree with the experimental data. The recast data collapse well onto the corresponding analytical solutions in Figure $3 \mathrm{~b}$. The larger oversaturation for bubble $\mathrm{B}$ is attributed to the nonuniform distribution of gas concentration in space. The smaller oversaturation for bubble $\mathrm{C}$ can be explained by a lower saturation level, due to an expiration of the catalytic reaction (see Figure 4). 
The end of each data set in Figure 2 and Figure 3 represents the detachment of the bubble. The size of the bubble at detachment is related to the size $R_{\mathrm{p}}$ of the bubble base pinning area (see sketch in Figure $5 b$ ) and had been predicted from balancing capillarity with buoyancy, ${ }^{24}$ giving the so-called Fritz radius

$$
R_{\mathrm{F}}=\left(\frac{3}{2} \frac{\sigma R_{\mathrm{p}}}{\rho_{\mathrm{L}} g}\right)^{1 / 3}
$$

Here $\sigma=0.074 \mathrm{~N} \cdot \mathrm{m}^{-1}$ and $\rho_{\mathrm{L}}=1.11 \times 10^{3} \mathrm{~kg} \cdot \mathrm{m}^{-3}$ are respectively the surface tension and the density of $30 \%(\mathrm{w} / \mathrm{w})$ $\mathrm{H}_{2} \mathrm{O}_{2}$ solution. ${ }^{39,40}$

Figure 5a shows the experimental detaching bubble radius $R_{\mathrm{d}}$ as a function of the effective base radius $R_{\mathrm{p}}$. The experimental data follow the same scaling $R_{\mathrm{d}} \propto R_{\mathrm{p}}^{1 / 3}$ as the theoretical prediction for the Fritz radius $R_{\mathrm{F}} \propto R_{\mathrm{p}}^{1 / 3}$ but is about $20 \%$ lower in value. That the Fritz radius can only be seen as an upper bound is not uncommon; see refs 32 and 41 . Here we also speculate that the discrepancy is caused by the buoyancyinduced necking distortion of the bubble root immediately before the detachment. In order to verify this speculation, we zoomed into the root portion of one bubble to observe the morphological change of the liquid-gas interface in detail. Three-dimensional confocal images exhibiting the variation were taken using a $60 \times$ oil immersion objective (CFI Apo Lambda S $60 \times$ Oil/1.4 DIC, NA $=1.4, \mathrm{WD}=0.14 \mathrm{~mm}$ ) to capture the details around the bubble root by recording the reflection signals. The cross-sectional images were extracted to show the geometrical variation of the bubble root as a function of time, and a corresponding movie is included in the Supporting Information. The inset of Figure 5a is the snapshot of the bubble root immediately before detaching. It is clearly illustrated that the bubble surface is subject to a necking stretch and the contact angle $\theta$ (defined in Figure $5 \mathrm{~b}$ ) substantially increases so that the surface tension can resist the buoyancy to postpone the detachment furthest. Note that the detaching behavior of a bubble forming on a flat substrate here is different from that growing on a thin needle from which eq 8 is derived. For the latter detachment, ${ }^{24}$ bubbles always pin at the singularity of the needle tip, resulting in that the capillarity force is approximately in the opposite direction to the buoyancy force, i.e., $\theta \approx 90^{\circ}$ immediately before detachment, while for the former case, the relationship between the interfacial tensions of liquid-gas, gas-solid, and solid-liquid at the contact line should also be considered according to the wetting properties of the substrate. Therefore, the theoretical prediction needs to be modified to take into account the influence of the change of $\theta$. Thus, by balancing the buoyancy force $F_{\mathrm{b}}=(4 /$ 3) $\pi R^{3} \rho_{\mathrm{L}} g$ and the vertical component of the capillarity force $F_{\mathrm{c} \perp}$ $=2 \pi R_{\mathrm{p}} \sigma \sin \theta$, eq 8 is revised to a modified Fritz radius

$$
R_{\mathrm{F}}^{\prime}=\left(\frac{3}{2} \frac{\sigma R_{\mathrm{p}} \sin \theta}{\rho_{\mathrm{L}} g}\right)^{1 / 3}
$$

For the measured value $\theta=38^{\circ}$, the modified Fritz radius is plotted as the dotted line in Figure 5a, which has a better agreement with the experimental data. This value is consistent with the contact angle of $41 \pm 6^{\circ}$ through measuring a $\mathrm{H}_{2} \mathrm{O}_{2}$ droplet on the gold substrate which has been submerged into $30 \%$ (w/w) $\mathrm{H}_{2} \mathrm{O}_{2}$ solution for $1 \mathrm{~h}$ beforehand.

\section{CONCLUSIONS}

In conclusion, bubble formation due to catalytic reactions has been experimentally and theoretically examined. We performed confocal microscopy to observe the growth of oxygen bubbles during the gold-catalyzed decomposition of hydrogen peroxide. Both chemical catalysis and physical mass transfer influence the dynamical evolution of bubbles.

First, we find that the bubble growth is limited by gas diffusion rather than the chemical reaction, which should also hold for the other common catalysts with lower activation energies than gold, as long as $\mathrm{Da}>1$. This diffusion-limited process ensures a supersaturation environment, providing a necessary condition for the nucleation and growth of bubbles which benefits gas-evolution relevant applications like selfpropulsion of micromotors.

Second, a balance can be reached between gas production from the catalytic reaction and diffusive gas supply to the bubble growth, leading to a well-repeated growing behavior for the closely consecutive bubbles, while spatially or temporarily inhomogeneous catalytic activity will cause the differences in the local gas oversaturation, resulting in substantial discrepancies in growth behaviors for the bubbles far apart from each other either in space or in time. The growing and detaching behaviors have significant effects on the working performances in practical applications, such as the speed and moving direction of self-propulsion and the efficiency and homogeneity of mass and heat transfer, through bubble growth rate, distributed nucleation, detaching size and frequency, and so on. For example, the well-repeated growing and detaching behaviors will generate a lasting constant recoil force exerted on the motor, leading to a forward movement in a single direction, while the spatially discrepant growth and detachment will cause an inhomogeneous force distribution on the motor, which consequently results in a rotation movement.

Finally, analyses show that in order to predict the bubble size at the moment of detachment more accurately, the detailed bubble geometry at the contact line must be taken into account. This work has thus improved the fundamental understanding of the diffusive bubble growth in a catalytic reaction, which is the basis for revealing mechanisms of many applications related to gas-evolving reactions. The mechanism reported in this work can provide valuable guidelines for design of reaction conditions to facilitate removal of bubbles from the substrate and mass and heat transfer through bubble detachment and transport.

\section{ASSOCIATED CONTENT}

\section{S Supporting Information}

The Supporting Information is available free of charge on the ACS Publications website at DOI: 10.1021/acs.jpcc.7b04994.

Movie showing the calculated concentration profile of $\mathrm{H}_{2} \mathrm{O}_{2}$ across the depth of the liquid over $5 \mathrm{~h}$ (AVI) Movie showing the calculated concentration profile of $\mathrm{O}_{2}$ across the depth of the liquid over $5 \mathrm{~h}$ (AVI)

Movie of the necking distortion of the bubble root immediately before the detachment (AVI)

Detailed calculations of Damköhler number and analyses of the concentration profile (PDF)

\section{AUTHOR INFORMATION}

\section{Corresponding Authors}

*E-mail: xuehua.zhang@rmit.edu.au. 
*E-mail: d.lohse@utwente.nl.

\section{ORCID $\odot$}

Pengyu Lv: 0000-0003-2265-4366

Xuehua Zhang: 0000-0001-6093-5324

Detlef Lohse: 0000-0003-4138-2255

\section{Notes}

The authors declare no competing financial interest.

\section{ACKNOWLEDGMENTS}

We thank G. Mul, E. Dietrich, Y. Yang, and Á. Moreno Soto for the helpful discussions and comments on the ar ticle. This work was supported by The Netherlands Center for Multiscale Catalytic Energy Conversion (MCEC), an NWO Gravitation programme funded by the Ministry of Education, Culture and Science of the government of The Netherlands. X.Z. acknowledges support from the Australian Research Council (FT120100473, DP140100805, and LP140100594).

\section{REFERENCES}

(1) Whitney, G. M.; Tobias, C. W. Mass-Transfer Effects of Bubble Streams Rising near Vertical Electrodes. AIChE J. 1988, 34, 19811995.

(2) Vogt, H.; Stephan, K. Local Microprocesses at Gas-Evolving Electrodes and Their Influence on Mass Transfer. Electrochim. Acta 2015, 155, 348-356.

(3) Volanschi, A.; Olthuis, W.; Bergveld, P. Gas Bubbles Electrolytically Generated at Microcavity Electrodes used for the Measurement of the Dynamic Surface Tension in Liquids. Sens. Actuators, A 1996, $52,18-22$.

(4) Huang, W.; Manjare, M.; Zhao, Y. Catalytic Nanoshell Micromotors. J. Phys. Chem. C 2013, 117, 21590-21596.

(5) Wang, H.; Zhao, G.; Pumera, M. Beyond Platinum: BubblePropelled Micromotors based on $\mathrm{Ag}$ and $\mathrm{MnO} 2$ Catalysts. J. Am. Chem. Soc. 2014, 136, 2719-2722.

(6) Pinchasik, B.-E.; Möhwald, H.; Skirtach, A. G. Mimicking Bubble Use in Nature: Propulsion of Janus Particles due to HydrophobicHydrophilic Interactions. Small 2014, 10, 2670-2677.

(7) Li, J.; Huang, G.; Ye, M.; Li, M.; Liu, R.; Mei, Y. Dynamics of Catalytic Tubular Microjet Engines: Dependence on Geometry and Chemical Environment. Nanoscale 2011, 3, 5083-5089.

(8) Guix, M.; Mayorga-Martinez, C. C.; Merkoci, A. Nano/ Micromotors in (Bio)chemical Science Applications. Chem. Rev. 2014, 114, 6285-6322.

(9) Wang, S.; Wu, N. Selecting the Swimming Mechanisms of Colloidal Particles: Bubble Propulsion versus Self-Diffusiophoresis. Langmuir 2014, 30, 3477-3486.

(10) Paxton, W. F.; Kistler, K. C.; Olmeda, C. C.; Sen, A.; St. Angelo, S. K., St; Cao, Y. Y.; Mallouk, T. E.; Lammert, P. E.; Crespi, V. H. Catalytic Nanomotors: Autonomous Movement of Striped Nanorods. J. Am. Chem. Soc. 2004, 126, 13424-13431.

(11) Gibbs, J. G.; Zhao, Y.-P. Autonomously Motile Catalytic Nanomotors by Bubble Propulsion. Appl. Phys. Lett. 2009, 94, 163104.

(12) Čolić, V.; Tymoczko, J.; Maljusch, A.; Ganassin, A.; Schuhmann, W.; Bandarenka, A. S. Experimental Aspects in Benchmarking of the Electrocatalytic Activity. ChemElectroChem 2015, 2, 143-149.

(13) Hernández, S.; Barbero, G.; Saracco, G.; Alexe-Ionescu, A. Considerations on Oxygen Bubble Formation and Evolution on BiVO4 Porous Anodes Used in Water Splitting Photoelectrochemical Cells. J. Phys. Chem. C 2015, 119, 9916-9925.

(14) Suslick, K. S.; Price, G. J. Applications of Ultrasound to Materials Chemistry. Annu. Rev. Mater. Sci. 1999, 29, 295-326.

(15) Harvey, E. N.; Barnes, D. K.; McElroy, W. D.; Whiteley, A. H.; Pease, D. C.; Cooper, K. W. Bubble Formation in Animals. I. Physical Factors. J. Cell. Comp. Physiol. 1944, 24, 1-22.

(16) Atchley, A. A.; Prosperetti, A. The Crevice Model of Bubble Nucleation. J. Acoust. Soc. Am. 1989, 86, 1065-1084.
(17) Caupin, F.; Herbert, E. Cavitation in Water: a Review. C. $R$. Phys. 2006, 7, 1000-1017.

(18) Lohse, D.; Prosperetti, A. Homogeneous Nucleation: Patching the Way from the Macroscopic to the Nanoscopic Description. Proc. Natl. Acad. Sci. U. S. A. 2016, 113, 13549-13550.

(19) Lohse, D.; Zhang, X. Surface Nanobubbles and Nanodroplets. Rev. Mod. Phys. 2015, 87, 981-1035.

(20) Epstein, P. S.; Plesset, M. S. On the Stability of Gas Bubbles in Liquid-Gas Solutions. J. Chem. Phys. 1950, 18, 1505-1509.

(21) Scriven, L. E. On the Dynamics of Phase Growth. Chem. Eng. Sci. 1959, 10, 1-13.

(22) Favelukis, M.; Yablonsky, G. S. Catalytic Bubble Model: Bubble Growth with an Interfacial Chemical Reaction. Ind. Eng. Chem. Res. 2004, 43, 4476-4482.

(23) Enríquez, O. R.; Sun, C.; Lohse, D.; Prosperetti, A.; van der Meer, D. The Quasi-Static Growth of $\mathrm{CO}_{2}$ Bubbles. J. Fluid Mech. 2014, 741, R1.

(24) Oguz, H. N.; Prosperetti, A. Dynamics of Bubble-Growth and Detachment from a Needle. J. Fluid Mech. 1993, 257, 111-145.

(25) Jones, S. F.; Evans, G. M.; Galvin, K. P. The Cycle of Bubble Production from a Gas Cavity in a Supersaturated Solution. Adv. Colloid Interface Sci. 1999, 80, 51-84.

(26) Barker, G. S.; Jefferson, B.; Judd, S. J. The Control of Bubble Size in Carbonated Beverages. Chem. Eng. Sci. 2002, 57, 565-573.

(27) Nahra, H. K.; Kamotani, Y. Prediction of Bubble Diameter at Detachment from a Wall Orifice in Liquid Cross-Flow under Reduced and Normal Gravity Conditions. Chem. Eng. Sci. 2003, 58, 55-69.

(28) Peñas-López, P.; Parrales, M. A.; Rodriguez-Rodriguez, J. Dissolution of a $\mathrm{CO} 2$ Spherical Cap Bubble Adhered to a Flat Surface in Air-Saturated Water. J. Fluid Mech. 2015, 775, 53-76.

(29) Chu, S.; Prosperetti, A. History Effects on the Gas Exchange between a Bubble and a Liquid. Phys. Rev. Fluids 2016, 1, 064202.

(30) Peñas-López, P.; Soto, Á. M.; Parrales, M. A.; van der Meer, D.; Lohse, D.; Rodríguez-Rodríguez, J. The History Effect on Bubble Growth and Dissolution. Part 2. Experiments and Simulations of a Spherical Bubble Attached to a Horizontal Flat Plate. J. Fluid Mech. 2017, 820, 479-510.

(31) Peñas-López, P.; Parrales, M. A.; Rodriguez-Rodriguez, J.; van der Meer, D. The History Effect in Bubble Growth and Dissolution. Part 1. Theory. J. Fluid Mech. 2016, 800, 180-212.

(32) Moreno Soto, Á.; Prosperetti, A.; Lohse, D.; van der Meer, D. J. Fluid Mech., to be submitted.

(33) McKee, D. W. Catalytic Decomposition of Hydrogen Peroxide by Metals and Alloys of the Platinum Group. J. Catal. 1969, 14, 355364.

(34) Goszner, K.; Bischof, H. The Decomposition of Hydrogen Peroxide on Silver-Gold Alloys. J. Catal. 1974, 32, 175-182.

(35) Eley, D. D.; MacMahon, D. M. The Decomposition of Hydrogen Peroxide Catalysed by Palladium-Gold Alloy Wires. J. Colloid Interface Sci. 1972, 38, 502-510.

(36) Goldstein, J. R.; Tseung, A. C. C. The Kinetics of Hydrogen Peroxide Decomposition Catalyzed by Cobalt-Iron Oxides. J. Catal. 1974, 32, 452-465.

(37) Watanabe, H.; Iizuka, K. The Influence of Dissolved Gases on the Density of Water. Metrologia 1985, 21, 19-26.

(38) Sander, R. Compilation of Henry's Law Constants (version 4.0) for Water as Solvent. Atmos. Chem. Phys. 2015, 15, 4399-4981.

(39) Phibbs, M. K.; Giguere, P. A. Hydrogen Peroxide and its Analogues. 1. Density, Refractive Index, Viscosity, and Surface Tension of Deuterium Peroxide Deuterium - Oxide Solutions. Can. J. Chem. 1951, 29, 173-181.

(40) Easton, M. F.; Mitchell, A. G.; Wynne-jones, W. F. K. The Behaviour of Mixtures of Hydrogen Peroxide and Water. 1. Determination of the Densities of Mixtures of Hydrogen Peroxide and Water. Trans. Faraday Soc. 1952, 48, 796-801.

(41) Simmons, J. A.; Sprittles, J. E.; Shikhmurzaev, Y. D. The Formation of a Bubble from a Submerged Orifice. Eur. J. Mech. BFluids 2015, 53, 24-36. 